\title{
Determining Position and Orientation of a 3- Wheel Robot on a Pipe Using an Accelerometer
}

\author{
Alexander McGregor ${ }^{1}$, Gordon Dobie ${ }^{1}$, Neil Pearson ${ }^{2}$, Charles MacLeod ${ }^{1}$, Anthony Gachagan ${ }^{1}$ \\ ${ }^{1}$ University of Strathclyde, Glasgow, G1 1XQ, Scotland, UK \\ ${ }^{2}$ Eddyfi Technologies, Swansea, SA6 8QY, Wales, UK
}

\begin{abstract}
Accurate positioning of robots on pipes is a challenge in automated industrial inspection. It is typically achieved using expensive and cumbersome external measurement equipment. This paper presents an Inverse Model method for determining the orientation angle $(\alpha)$ and circumferential position angle $(\omega)$ of a 3 point of contact robot on a pipe where measurements are taken from a 3-axis accelerometer sensor. The advantage of this system is that it provides absolute positional measurements using only a robot mounted sensor. Two methods are presented which follow an analytical approximation to correct the estimated values. First, a correction factor found though a parametric study between the robot geometry and a given pipe radius, followed by an optimization solution which calculates the desired angles based on the system configuration, robot geometry and the output of a 3-axis accelerometer. The method is experimentally validated using photogrammetry measurements from a Vicon T160 positioning system to record the position of a three point of contact test rig in relation to a test pipe in a global reference frame. An accelerometer is attached to the 3 point of contact test rig which is placed at different orientation $(\alpha)$ and circumferential position $(\omega)$ angles. This work uses a new method of processing data from an accelerometer sensor to obtain the $\alpha$ and $\omega$ angles. The experimental results show a maximum error of $3.40^{\circ}$ in $\alpha$ and $4.17^{\circ}$ in $\omega$, where the $\omega$ circumferential positional

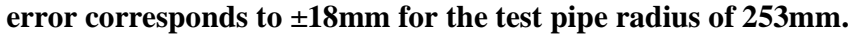

Index Terms-Accelerometer, Crawler, IMU, Mobile, NDE, NDT, Pipe Inspection, Positioning

\section{INTRODUCTION}

$\mathrm{O}$ any given industrial site in the petrochemicals supply chain there are components which need to be routinely inspected to detect and monitor defects such as cracks or loss in wall thickness. Non-Destructive Evaluation (NDE) allows for a component to be inspected non-intrusively and is necessary to ensure the integrity of a structure. Inspections are usually performed manually, where an operator has to move the sensor

\footnotetext{
${ }^{T}$ he authors would like to thank the RCNDE and Eddyfi Technologies for their financial support under Grant EP/L015587/1.

A. McGregor is with the University of Strathclyde at the Centre for Ultrasonic Engineering (CUE), Glasgow, G1 1RD, Glasgow, Scotland (email: alec.mcgregor@strath.ac.uk)

G. Dobie is with the University of Strathclyde at the Centre for Ultrasonic Engineering (CUE), Glasgow, G1 1RD, Glasgow, Scotland (email: gordon.dobie@strath.ac.uk)

N. R. Pearson is with Eddyfi Technologies at Eddyfi UK, SA6 8QY, Swansea, Wales (email: n.pearson@eddyfi.com)
}

by hand, which can lead to measurement errors from inspector fatigue and operator bias [1]. This can cause defects to be missed or misrepresented by being mapped in the wrong place. Inadequate NDE can potentially lead to the catastrophic failure of a component which can have severe safety, operational and financial implications.

Pipelines are commonly inspected in the petrochemicals industry. On any given site there could be hundreds of kilometres of pipes which need to be monitored. Locating and sizing defects in pipes allows asset owners to maximise the lifetime of the pipe while removing the need for unnecessary repairs and downtime. Accuracy and precision of defect localisation is critical as it can reduce the overall time taken for the maintenance process by increasing repair accuracy and ascertaining the correct location for monitoring defect growth through subsequent inspections.

Using an automated system can increase precision and accuracy of defect localisation while omitting the typical disadvantages associated with human operator inconsistency [2]. Automated inspection with robotics is playing an increased role in industry as automation can increase the inspection speed, decrease risk to operator health and safety and can have financial benefits in terms of reduced training and site preparation costs.

While there are many benefits from implementing robotic solutions, there are still challenges to overcome. One key problem associated with mobile robotics and defect mapping is obtaining an accurate spatial position of the NDE measurement in a cost-effective manner over long periods of time. Errors in these measurements are due to the inherent variability of positioning sensors.

Positioning systems exist which can accurately measure the position and orientation of an object in 3D space with millimetre accuracy, such as photogrammetry [3] and LiDar systems [4]. These measure absolute position in a global reference frame which means that the error associated with the measurement remains constant with time. They are usually

C. MacLeod is with the University of Strathclyde at the Centre for Ultrasonic Engineering (CUE), Glasgow, G1 1RD, Glasgow (email: charles.macleod@strath.ac.uk)

A. Gachagan is with the University of Strathclyde at the Centre for Ultrasonic Engineering (CUE), Glasgow, G1 1RD, Glasgow (email: anthony.gachagan@strath.ac.uk) 
placed external from the robot, require extra site preparation, operator training and are costly. Practically, these can be difficult to deploy, require significant training and long set-up times. These systems also require line of sight, which becomes an issue when attempting to achieve full coverage of an asset such as a pipe.

An alternative to using external sensors is to use onboard sensors where there is no exterior equipment required. This reduces the amount of work needed to setup and prepare the inspection environment and can therefore reduce the overall time for inspection. One of the main disadvantages of onboard sensors is that they tend to use relative measurements and therefore have time integral error. This becomes a significant problem for continuous periods of inspection using a mobile or autonomous system as the positioning error will become greater over time.

It is possible to inspect a pipe from the inside or the outside. Inner inspection can require pipes to be isolated, emptied and cleaned which increases total inspection time. Outer pipe inspection can be quicker, however there are other challenges, including inspection of areas under supports and saddle welds [5].

Three-wheeled mobile crawler robots are becoming increasingly used in the inspection industry due to their advantages over conventional 4-wheeled robots [6]. These are namely; increased manoeuvrability, more simple kinematics compared to other wheeled robot types and more accurate dead reckoning, which is not feasible for 4-wheel designs due to wheel slippage [7] [8].

This paper describes a method of measuring the position and orientation of a 3- wheeled mobile robot on the outer surface of a horizontal pipe by calculating the circumferential angle $(\omega)$ and the orientation $(\alpha)$, shown in Fig. 1. This is achieved using a relatively low cost, onboard acceleration sensor and knowledge of both the pipe and robot geometry to produce an absolute measurement of these angles without time integral error.
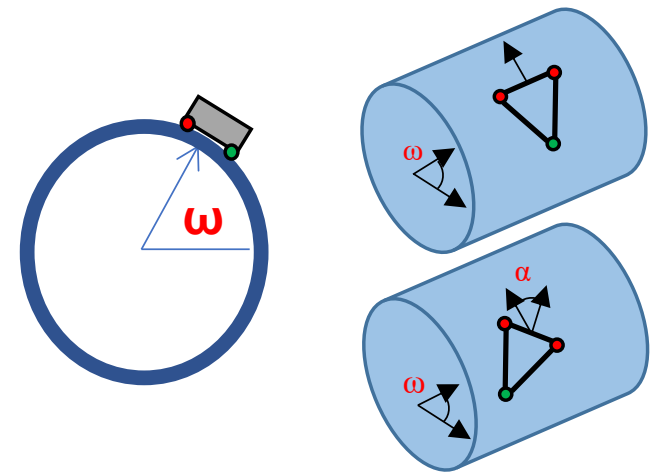

Fig. 1. Robot pipe localisation problem schematic depicting the position angle on the circumference of the pipe $(\omega)$ and the orientation angle $(\alpha)$ of a 3-wheeled robot on a horizontal pipe

\section{BACKGROUND AND LITERATURE}

\section{A. Positioning and Sensors}

There are two main types of positioning sensors and two main types of position measurements. These are external/internal sensors and absolute/relative measurements respectively [9], [10].
External Sensors: Sensors which are not attached to the robot body. Examples include GPS, LiDar and photogrammetry systems. These tend to be absolute measurements which is a measure of the position within a fixed global reference frame. The main disadvantages are that external sensors require increased set-up time, tend to be costlier and are bulky due to the extra external equipment required.

Onboard Sensors: Sensors which are attached to the robot. These tend to be relative measurements which rely on the previous measurements to obtain the current estimate and are therefore subject to time dependant integral error that increases over time. Examples include encoders and gyroscopes. They can be very accurate over short periods of time and do not require external apparatus to be set-up. Traditionally, using accelerometers for positioning incurs un unworkable integral error as the acceleration signal is double integrated to obtain a change in position.

\section{B. Inertial Measurement Unit}

An Inertial Measurement Unit (IMU) has become an affordable and viable onboard robotic sensor. This is due to advances in Micro-Electro-Mechanical Systems (MEMS) manufacture reducing manufacturing cost and size of the component [11].

IMUs can be used to estimate the orientation, velocity or position of a robot. A typical set-up for 9 Degree of Freedom (DOF) IMU is a 3-axis accelerometer, a 3 axis gyroscope and a 3 -axis magnetometer. An IMU sensor is a proprioceptor, it senses changes within the robotic system rather than sensing changes or movement from the outside world. This reduces the inaccuracies that are associated with the surrounding environment which occur with exteroceptive (external sensing) sensors such as ultrasonic and laser range finders.

IMUs are good at determining the orientation of an object. However, they are not very reliable at determining displacement due to the double integration of accelerometer readings and therefore is subject to integral error [12] [13]. Orientation estimation is usually achieved by fusing outputs of a 9 DOF IMU. However, in many industrial environments, the magnetic field vector cannot be taken as constant as there are local ferrous objects which may interfere with the local magnetic field [14], as well as magnetic interference from permanent magnets and magnetic wheels from a climbing robot. Gyroscopes are also subject to integral error as the angular acceleration is integrated to determine the angular change. Previous works have used a 3-axis accelerometer for tilt sensing of a stationary object which, has no drift over time due to there being no integration step [15] and relies on the 3axis accelerometer output only. However, this only gives the roll and pitch of the accelerometer, and the yaw component is necessary to calculate the orientation of the robot using this method. Due to this constraint using calculating tilt angles, another method of obtaining the orientation and position of the robot while only using accelerometer readings has been developed in this work. In this setup, the acceleration force due to gravity is taken to be a constant value in a constant direction which does not change with time. For the reasons mentioned 
above, this work only utilizes the 3 -axis accelerometer output from an IMU to calculate $\omega$ and $\alpha$ angles.

In industry, IMUs are used for both orientation and position estimation. IMU's are currently used as part of a localisation system. However, they are not used specifically for localisation on pipe surfaces. Examples of IMU's utilized in positioning include [16] [17] [18], where accelerometer data is used to determine distance travelled. However due to the relative measurement, these works include a correction method which attempts to reduce drift. This correction is usually a post capture filter in the form of a Kalman filter [19] or Particle Filter [20] which are common data fusion algorithms used in off the shelf IMUs and general robotics. Though not investigated here, they will be considered in future work. This work only considers the accelerometer output of an IMU.

\section{Current Pipe Inspection Systems}

Current mobile robotic systems on the market tend to rely on high resolution encoder measurements or other high cost sensors, such as photogrammetry or Lidar, rather than using costly external positioning systems. One example of this is the Silverwing RMS2 system. The RMS2 is a remote access corrosion mapping system and can be used to inspect storage tanks, pressure vessels and pipes [21]. Using encoders for short periods of time allows for accurate and precise localisation on a pipe, provided that the initial starting position is known.

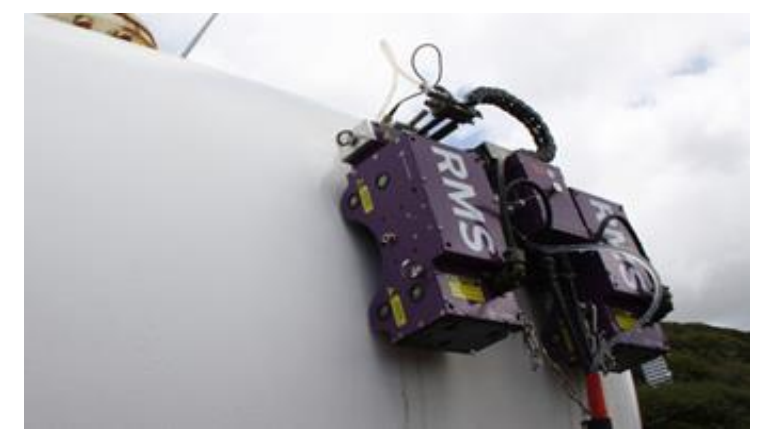

Fig. 2. Eddyfi Technologies Silverwing RMS2 inspecting a pipe using encoders for positional information for defect mapping

Another method of inspecting pipes is through manual devices such as the Eddyfi PEC (Pulsed Eddy Current) probe [22]. This method requires an operator to move the probe by hand. This entails drawing a grid on the pipe surface so that a corrosion map can be created and operators can determine which areas have been inspected, shown in Fig. 3. The gridding process is preparatory work which needs to be carried out prior to inspection and increases the overall inspection time. Incomplete or patchy defect maps are likely due to inconsistent scanning which can become more prevalent with operator fatigue [1].

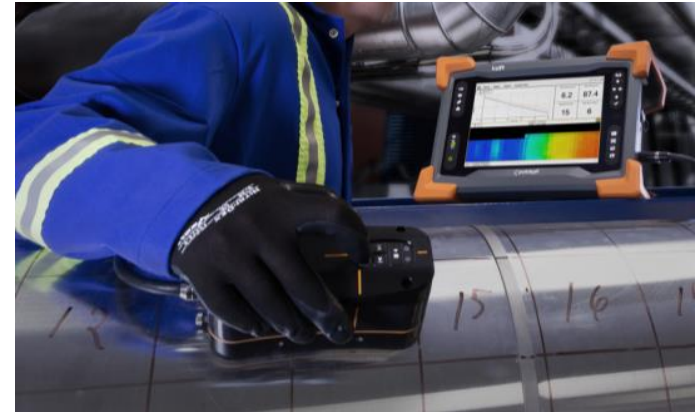

Fig. 3. Eddyfi PEC Probe with gridded pipe

Some inspection methods require highly accurate circumferential positional measurements. For such methods, track systems which are attached around the pipe are deployed and an example of a track is shown in Fig. 4. One advantage of this setup is that the angular position can be easily determined as the step size around the track is pre-set. Disadvantages with this system includes setup time and the system requires operators to move the whole system in order to scan a different section of pipe.

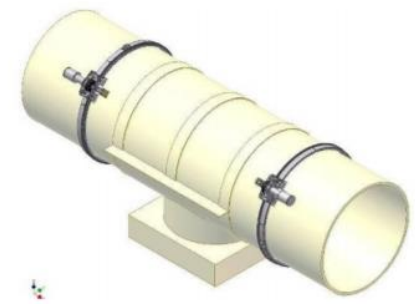

Fig. 4. Sonomatic track system for inspection of pipe supports[23]

\section{APPROACH}

To overcome the restrictions in the described applications, the proposed solution is to use a 3-axis accelerometer to measure the gravity vector. From only the accelerometer data, the orientation of the plane of the robot in 3D space can be determined. This plane orientation can then be fused with information about the geometry of the pipe and the robot to calculate the $\alpha$ and $\omega$ angles. These can then be used to calculate the 3 points of contact between the wheels of the robot and the pipe.

The required geometry information is the distance between the robot drive wheels (b), the length from the drive wheel axis and the castor wheel (l) and the radius of the pipe (r). The relationship between these variables is shown in Fig. 5.

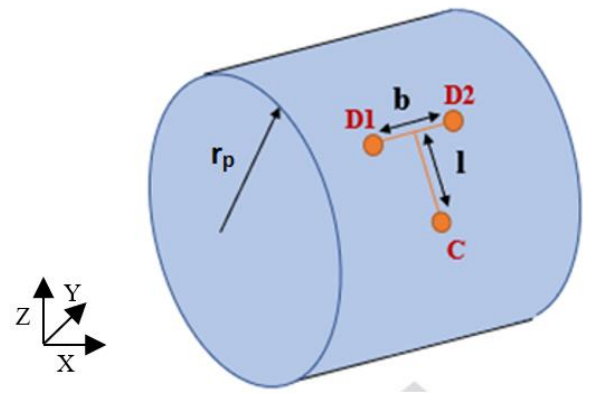

Fig. 5. Describing the required geometry knowledge for the proposed algorithm. $b$ is the distance between the two drive wheels (D1 and D2), 1 is the distance between the drive wheel axis and the castor wheel (C) and R is the radius of the pipe. 
Assumptions made for this work are:

- Gravity vector is taken as a constant reference, always acting downwards in the global reference frame

- Pipe is perfectly cylindrical with no grooves or ovality

- Only horizontal pipes are considered

\section{A. System Model}

The following series of 9 simultaneous equations (1)-(9) describe the system, linking the XYZ output of the accelerometer with the 3 points of contact on the pipe surface. It is not practical to solve these directly using the Gröbner basis [24] as the result was a polynomial with an order in excess of 100. However, they are included here for completeness.

$$
\begin{gathered}
\left(Y_{D 1}+Y_{D 2}+Y_{C}\right) \frac{1}{3}=0 \\
X_{D 1}^{2}+Z_{D 1}^{2}=R^{2} \\
X_{D 2}^{2}+Z_{D 2}^{2}=R^{2} \\
X_{C}^{2}+Z_{C}^{2}=R^{2} \\
\left(X_{D 1}-X_{D 2}\right)^{2}+\left(Y_{D 1}-Y_{D 2}\right)^{2}+\left(Z_{D 1}-Z_{D 2}\right)^{2}=b^{2} \\
\left(\frac{1}{2} X_{D 1}+\frac{1}{2} X_{D 2}-X_{C}\right)^{2}+\left(\frac{1}{2} Y_{D 1}+\frac{1}{2} Y_{D 2}-Y_{C}\right)^{2} \\
+\left(\frac{1}{2} Z_{D 1}+\frac{1}{2} Z_{D 2}-Z_{C}\right)^{2}=l^{2} \\
(\vec{D} 1-D 2) \cdot\left(\frac{D 1+D 2}{2}-C\right)=0 \\
n(1) X_{D 1}+n(2) Y_{D 1}+n(3) Z_{D 1}=n(1) X_{C}+n(2) Y_{C}+n(3) Z_{C} \\
n(2) Y_{D 2}+n(3) Z_{D 2}=n(1) X_{C}+n(2) Y_{C}+n(3) Z_{C}
\end{gathered}
$$

Where:

- Equation (1) sets the robots length along the pipe to 0

- Equations $(2-4)$ ensure that the points of contact lie on the curved surface of the pipe

- Equations $(5-7)$ set the geometry of the robot

- Equations $(8-9)$ ensures that all the points of contact lie on the same plane

- D1, D2 and C are the geometries illustrated in Fig. 5. $\mathrm{X}, \mathrm{Y}$ and $\mathrm{Z}$ are the coordinates taken from the centre of the pipe

- $\left(\mathrm{X}_{\mathrm{D} 1}, \mathrm{Y}_{\mathrm{D} 1}, \mathrm{Z}_{\mathrm{D} 1}\right),\left(\mathrm{X}_{\mathrm{D} 2}, \mathrm{Y}_{\mathrm{D} 2}, \mathrm{Z}_{\mathrm{D} 2}\right)$ and $\left(\mathrm{X}_{\mathrm{c}}, \mathrm{Y}_{\mathrm{c}}, \mathrm{Z}_{\mathrm{c}}\right)$ are the coordinates of the contact points and the variables to be solved for

- $\mathrm{n}$ is the unit vector normal to the plane of the accelerometer calculated from accelerometer readings

The method used in this work uses a forward/inverse model approach. A forward model is first created to describe the physical system and is used to simulate the accelerometer readings with given $\omega$ and $\alpha$ angles. An inverse model is then created to calculate the $\omega$ and $\alpha$ angles from the 3 axis accelerometer data. An optimisation approach is then used between the initial simulated accelerometer measurements and the simulated accelerometer measurements at the calculated $\alpha$ and $\omega$ angles. This process is outlined in Fig. 6 , where the optimised $\alpha$ and $\omega$ output should be equal to the initial $\alpha$ and $\omega$ input.

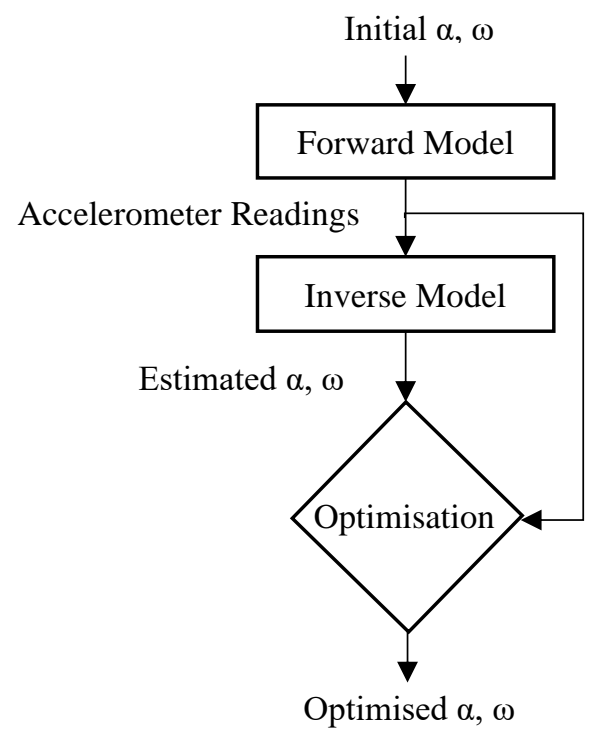

Fig. 6. Process flow diagram of method used to solve the problem.

\section{FORWARD MODEL}

The mathematical model used to determine the three contact points is based on the curves of intersection created by two perpendicular cylinders (front drive wheel positions) and the intersection between a sphere and a cylinder (back passive castor wheel position), where the centre of rotation is taken around the midpoint between the two drive wheels. The parametric equations used to calculate the drive wheel points on the curve of intersection of two cylinders are given by equations (10)-( 12). The parametric equations used to calculate the position of the castor wheel points is given by the equations of a sphere in equations (14)-(16).

$$
\begin{gathered}
X_{D}=\frac{b}{2} \sin (\alpha+(D-1) \pi) \\
Y_{D}=\frac{b}{2} \cos (\alpha+(D-1) \pi) \\
Z_{D}=\sqrt{\left\|R_{p}^{2}-X_{D}{ }^{2}\right\|} \\
X_{C}=l * \cos (\alpha) \sin (\gamma) \\
Y_{C}=l * \sin (\alpha) \sin (\gamma) \\
Z_{C}=l * \cos (\alpha) \sin (\gamma)+Y_{D}
\end{gathered}
$$

Where; $b$ is the wheelbase of the robot or the diameter of one of the intersection cylinders, $\alpha$ is the orientation angle of the robot, $\mathrm{D}$ is the drive wheel number being either 1 or $2, \mathrm{R}_{\mathrm{P}}$ is the radius of the pipe, 1 is the length of the robot or radius of the sphere, and $\gamma$ is the angle of elevation from the centre of the sphere. 
From the 3 contact points, a plane is created and the normal vector to the plane is found ( $\mathrm{Z}_{\mathrm{IMU}}$ direction). The $\mathrm{X}_{\mathrm{IMU}}$ direction is found by taking the direction from the centre of the drive wheels to the left drive wheel and the $\mathrm{Y}_{\text {IMU }}$ direction is the cross product of these two vectors. Gravity is taken to always be acting in the negative $\mathrm{Z}_{\text {Global }}$ direction. The outline is illustrated in Fig. 7.

Once all 3 accelerometer axis directions are determined, the gravity vector is separated into the 3 constituent vectors $\left(\mathrm{X}_{\mathrm{IMU}}\right.$, $\mathrm{Y}_{\mathrm{IMU}}$, and $\mathrm{Z}_{\mathrm{IMU}}$ ) by calculating the angle between the vectors [25]. Fig. 7 shows an example simulation with the robot reference frame $\left(\mathrm{X}_{\mathrm{IMU}}, \mathrm{Y}_{\mathrm{IMU}}\right.$, and $\left.\mathrm{Z}_{\mathrm{IMU}}\right)$ in relation to the global reference frame $\left(\mathrm{X}_{\mathrm{Global}}, \mathrm{Y}_{\mathrm{Global}}\right.$, and $\left.\mathrm{Z}_{\mathrm{Global}}\right)$ and gravity $(\mathrm{g})$.

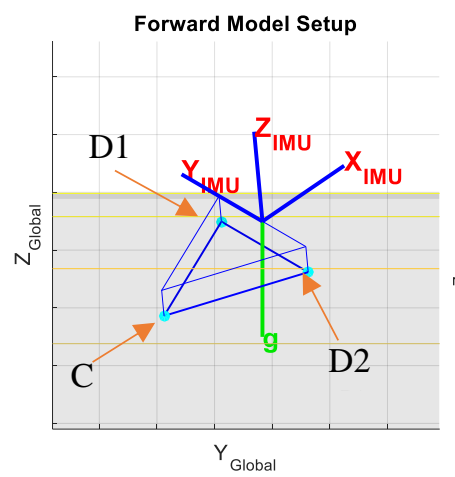

a)

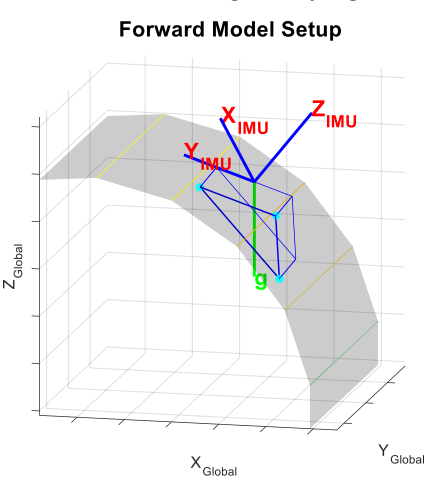

b)
Fig. 7. Example setup of IMU reference frame with respect to the global reference frame and gravity

\section{INVERSE MODEL}

The inverse model is not a direct inverse of the presented forward model. This is because there is no unique solution to find the direction of the accelerometer axes from the 3-axis accelerometer readings alone and the Inverse model is ill-posed. This is because there is only 1 equation, and 3 unknowns, as shown in (16), which represents a method of calculating the angle between two vectors (gravity vector and $\mathrm{X}$ accelerometer direction in this case), where:

- $\gamma_{g x}$ is the angle between the chosen accelerometer axis in a global reference frame (in this case the $\mathrm{X}$ direction) which can be calculated from the accelerometer readings

- $\mathrm{X}_{\mathrm{xg}, \mathrm{yg}, \mathrm{zg}}$ are the $\mathrm{x}, \mathrm{y}, \mathrm{z}$, components of the accelerometer $\mathrm{X}$ direction in terms of the global reference, which need to be calculated in the Inverse Model

- $\mathrm{g}_{\mathrm{xg}, \mathrm{yg}, \mathrm{zg}}$ are the $\mathrm{x}, \mathrm{y}, \mathrm{z}, \mathrm{components} \mathrm{of} \mathrm{the} \mathrm{gravity}$ direction in terms of the global reference, which are known.

$$
\gamma_{g x}=\cos ^{-1}\left(\frac{g_{x} x_{x}+g_{y} x_{y}+g_{z} x_{z}}{\sqrt{g_{x}^{2}+g_{y}^{2}+g_{z}^{2}} * \sqrt{x_{x}^{2}+x_{y}^{2}+x_{z}^{2}}}\right)
$$

Due to this, another method of calculating position and orientation angles was used. The simulated accelerometer readings $\left(\mathrm{A}_{\mathrm{x}}, \mathrm{A}_{\mathrm{Y}}\right.$ and $\left.\mathrm{A}_{\mathrm{z}}\right)$ are used to estimate the $\alpha$ and $\omega$ angles using (17) and (18).

$$
\begin{gathered}
\omega=\operatorname{asin}\left(\frac{A_{z}}{g}\right) \\
\alpha=\operatorname{atan} 2\left(A_{y}, A_{x}\right)
\end{gathered}
$$

Where;

- $\mathrm{A}_{\mathrm{x}}$ and $\mathrm{A}_{\mathrm{y}}$ and $\mathrm{A}_{\mathrm{z}}$ are the $\mathrm{X}, \mathrm{Y}$ and $\mathrm{Z}$ accelerometer readings in $\mathrm{ms}^{-2}$

- $\mathrm{g}$ is the acceleration due to gravity in $\mathrm{ms}^{-2}$.

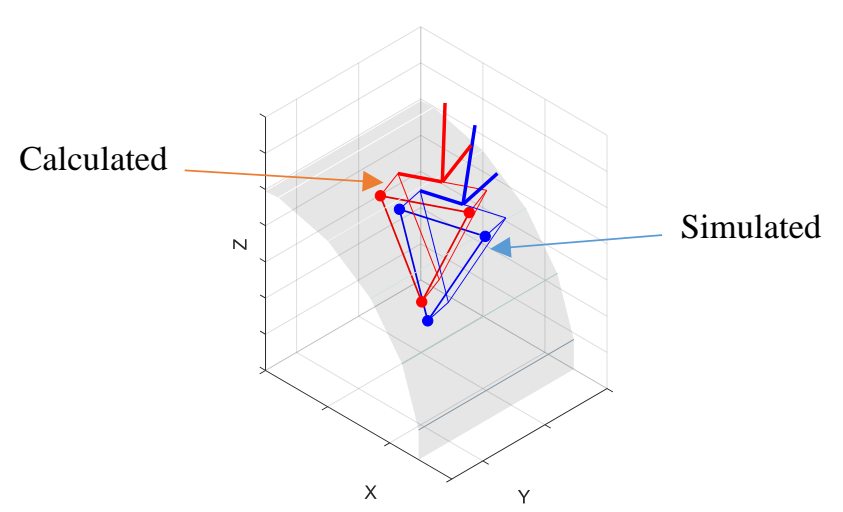

Fig. 8. Blue: simulated forward model position output. Red: output of equations (17) and (18) at estimated position

When the output of the above equations is plotted against the forward model, it is noticed that there is a significant error in the calculated angles compared to the simulated ones as seen in the example given in Fig. 8. This error is a result of (17) and (18) being approximations which do not take into account the change in pitch of the robot as it rotates in $\alpha$, which is a function of the geometry of the robot and the radius of the pipe. This error is present as the equations used in the Inverse model are only approximations and are used due to the ill-posed nature of the problem as mentioned previously. Two methods are presented to correct this error and compensate for the lack of a pure analytical solution; a parametric approach and an optimisation approach.

\section{A. Parametric Method}

A parametric investigation is conducted to determine the relationship between the error and the radius of the pipe at a fixed robot geometry. Fig. 9 shows an example of the oscillation in the $\omega$ angle as the robot is simulated to rotate $360^{\circ}$ in $\alpha$ at an $\omega$ position of $45^{\circ}$.

Fig. 10 shows the amplitude of oscillation in $\omega$ for different pipe radii simulated for the same $\alpha$ rotation and Fig. 11 shows the correction needed in the $\alpha$ angle to match the original input. 


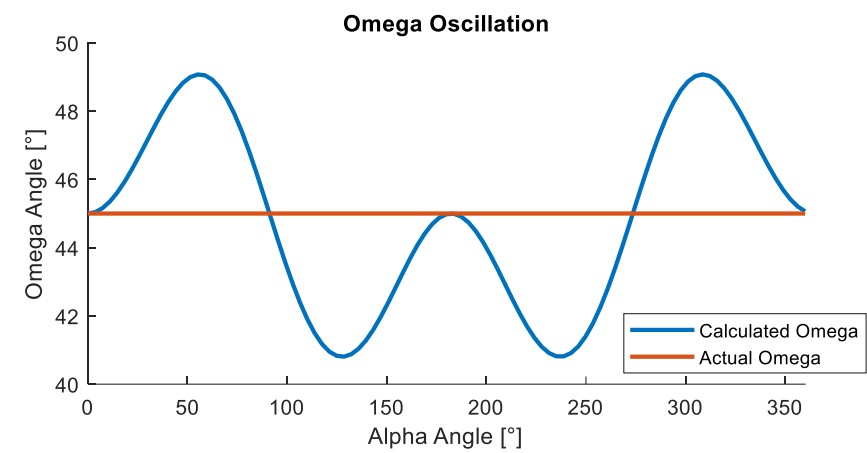

Fig. 9. Example showing discrepancy between the calculated $\omega$ (blue) and the simulated $\omega$ (red) while simulating a full $360^{\circ}$ rotation in the $\alpha$ angle

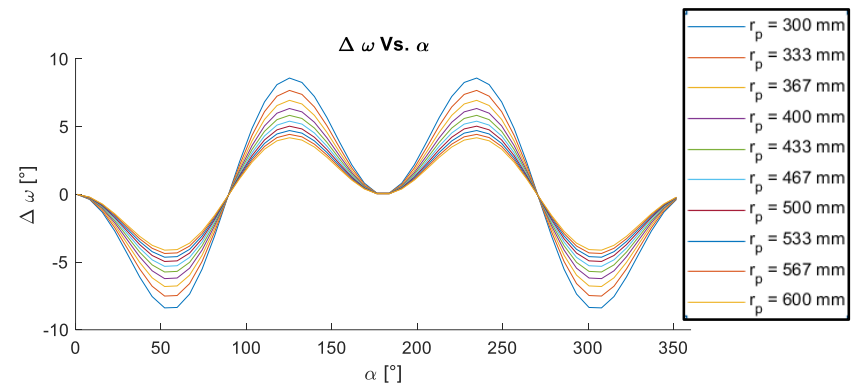

Fig. 10. Calculated $\omega$ values with respect to changing pipe radius $\left(r_{p}\right)$

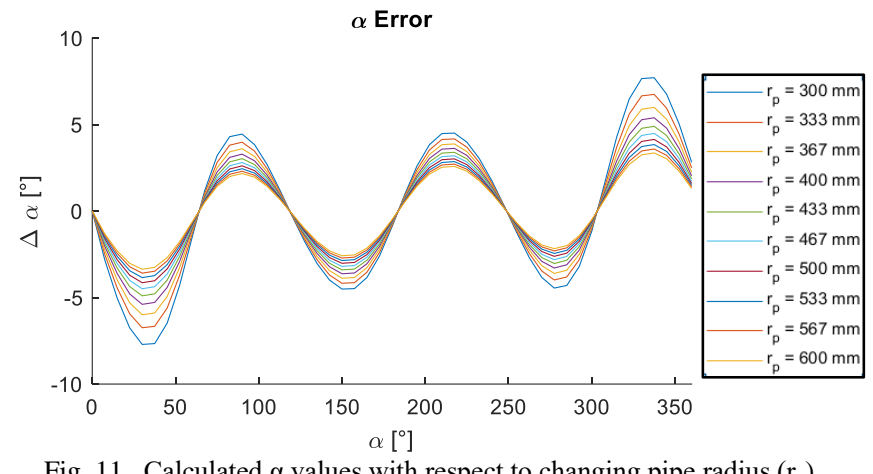

Fig. 11. Calculated $\alpha$ values with respect to changing pipe radius $\left(r_{p}\right)$

Equations (17) and (18) are modified to (19) and (20) where $\mathrm{C}_{1-4}$ are correction factors. These factors are calculated by using a line fitting function to determine the $\mathrm{C}$ values which will give the closest fit to the lines in Fig. 10 and Fig. 11 for each pipe radius tested and shows how the oscillation decreases as the pipe radius increases. A polynomial is then fitted to these values to produce an equation for $\mathrm{C}_{1}, \mathrm{C}_{2}, \mathrm{C}_{3}$ and $\mathrm{C}_{4}$ as a function of pipe radius $r_{p}$.

$$
\begin{gathered}
\omega_{\text {cor }}=\operatorname{atan} 2\left(\mathrm{~A}_{\mathrm{y}}, \mathrm{A}_{\mathrm{x}}\right)-\mathrm{C}_{1} \sin (\alpha) \sin (2 \alpha) \\
\alpha_{\text {cor }}= \\
\operatorname{atan} 2\left(\mathrm{~A}_{\mathrm{y}}, \mathrm{A}_{\mathrm{x}}\right)-\mathrm{C}_{2} \sin (3 * \alpha)+\mathrm{C}_{3} \cos \left(\mathrm{C}_{4} * \alpha\right)
\end{gathered}
$$

Fig. 12 a) shows the position of the robot calculated when using (20) and (19) compared to the simulated forward model position. Fig. $12 \mathrm{~b}$ ) shows the robot position corrected using the correction factor found using a parametric study, where a difference in the $\alpha$ angle can still be seen. Fig. $12 \mathrm{c}$ ) shows the comparison between the position calculated corrected for both the $\alpha$ and $\omega$ angles and the forward model. Fig. $12 \mathrm{c}$ ) shows that using the correction factors found using a parametric study can accurately correct the analytical approximations made using (20) and (19). It should be noted that Fig. 12 c) appears to show good agreement, there is a $<2^{\circ}$ error in both angles.

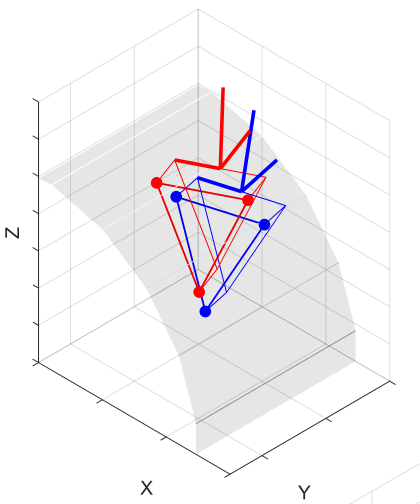

a)

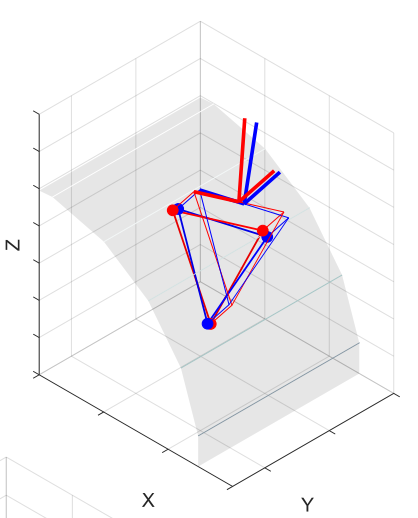

b)

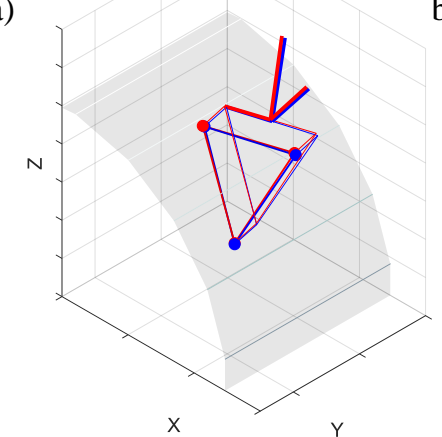

c)
Fig. 12. A comparison between the simulated robot position (Blue) and the output of the Inverse Model (Red). a) Uncorrected position b) $\omega$ correction c) $\omega$ and $\alpha$ correction

While this method is workable, it requires a parametric study to be completed for each robot/pipe geometry. In order to find a more general solution which holds for any reasonable robot geometry on any given pipe another method is considered.

\section{OPTIMISATION}

To overcome the discrepancy between the calculated and the simulated robot angles, an optimisation method is presented. A least mean squared error minimisation technique is used to achieve this. The steps taken are described as follows:

1. Calculate $\alpha_{\text {calc }}$ and $\omega_{\text {calc }}$ from accelerometer readings.

2. Simulate accelerometer readings from $\alpha_{\text {calc }}$ and $\omega_{\text {calc }}$ using the forward model.

3. Apply least mean squared error minimisation optimisation between the original accelerometer readings (Step 1.) and the simulated accelerometer (Step 2.) by varying $\alpha_{\text {calc }}$ and $\omega_{\text {calc }}$.

Equation (21) is the minimised cost function. The initial point of the optimisation are the values given by (17) and (18).

$$
f(\alpha, \omega)=\left(A_{x_{-} \_p t}-A_{x}\right)^{2}+\left(A_{\text {y_opt }}-A_{y}\right)^{2}+\left(A_{z_{-} \_p t}-A_{z}\right)^{2}
$$


This method returns an $\alpha$ and $\omega$ angle which outputs the same accelerometer data that are used in the forward model to simulate the corresponding acceleration readings.

\section{Simulation Vs. Algorithm Vs. Optimisation}

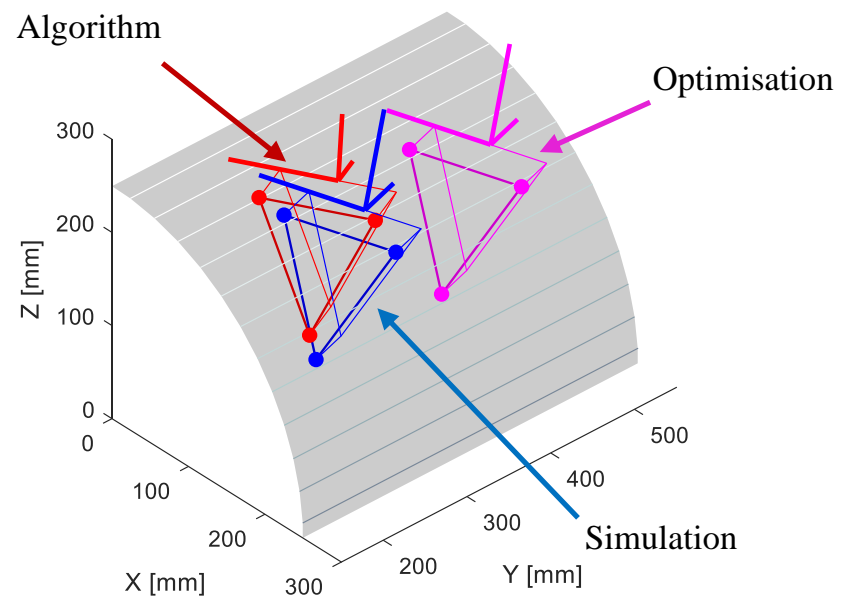

Fig. 13. Comparison between the simulated (blue), estimated (red) and optimized (pink) robot positions

A comparison between the simulated, calculated and optimised robot positions is shown in Fig. 13, where the optimised robot position is shifted along the $\mathrm{Y}$ axis. This is done as the optimised position is the same as the simulated position as seen with the values of the angles in TABLE I. This shows that in a perfect situation where the pipe is perfectly round and horizontal, a perfect accelerometer and flat robot, the optimisation solution converges to the exact angles.

TABLE I

Comparison between the simulated and calculated angles

\begin{tabular}{ccc}
\hline \hline & $\boldsymbol{\omega}$ & $\boldsymbol{\alpha}$ \\
\hline Simulation & $45.00^{\circ}$ & $45.00^{\circ}$ \\
Algorithm & $39.62^{\circ}$ & $39.07^{\circ}$ \\
Optimisation & $45.00^{\circ}$ & $45.00^{\circ}$ \\
\hline \hline
\end{tabular}

\section{DATA COLLECTION}

Experiments were conducted to check the validity of the mathematical models developed and to determine the suitability of this method for real world applications. A test rig was manufactured with 3 points of contact to simulate a 3 wheeled robot. The Vicon MX Giganet system utilising 12 Vicon T160 cameras was used to measure ground truth position and orientation of the test rig, as done in previous studies [26] [7] and was calibrated before use. The Vicon is a photogrammetry 6 DOF motion capture system which utilizes several cameras to track the position and orientation of an arrangement of retroreflective markers. Ring magnets were attached to the test rig to hold the rig in position on the metal pipe. XYZ positional data and the orientation in quaternions were recorded at a rate of $100 \mathrm{~Hz}$ for 3 seconds. The accelerometer on the IMU was sampled at a rate of $1 \mathrm{kHz}$ for 3 seconds. The IMU used in for this experiment an off the shelf XSens MTi-300. The sensitivity of the accelerometer is $40 \mathrm{mVg}^{-1}$ with the resolution being $0.0067 \mathrm{~ms}^{-2}$ Fig. 16 shows the manufactured test rig with the Vicon markers attached. A Leica laser measurement system [27] was used to test the accuracy of the accelerometer readings. The Leica system is a methodology based laser scanner which can measure the position of a retro reflector in free space to accuracies of $\pm 0.2 \mu \mathrm{m}$ [28]. The accuracy of the accelerometer readings was tested by using the Leica system to determine the angle of a wedge. The accelerometer was then placed on both sides of the wedge at different orientations and the roll and pitch were calculated from the accelerometer readings. The plane of the accelerometer on both surfaces is then calculated and the angle between the planes is taken as the wedge angle. The results showed a maximum discrepancy of $0.2^{\circ}$ which is in line with the accuracies stated in the datasheet. Two robot geometries were tested, both with a length (l) of $180 \mathrm{~mm}$ and the two wheel bases (b) being $180 \mathrm{~mm}$ and $70 \mathrm{~mm}$.

The test rig was placed at different stationary positions on the pipe with a nominal outer radius of $253.5 \mathrm{~mm}$ (nominally 10 ") by rotating the rig by hand and the data was recorded from the stationary test rig. Measurements were taken with the Vicon system to determine the ground truth position and orientation of the rig and with the acceleration recorded to calculate the position and orientation. The measurements were taken in a settled state. The experimental setup is shown in Fig. 14 where the pipe was placed in the centre of the measurement volume. Fig. 15 shows the pipe used for the experiment with the connecting pipe and saddle weld.

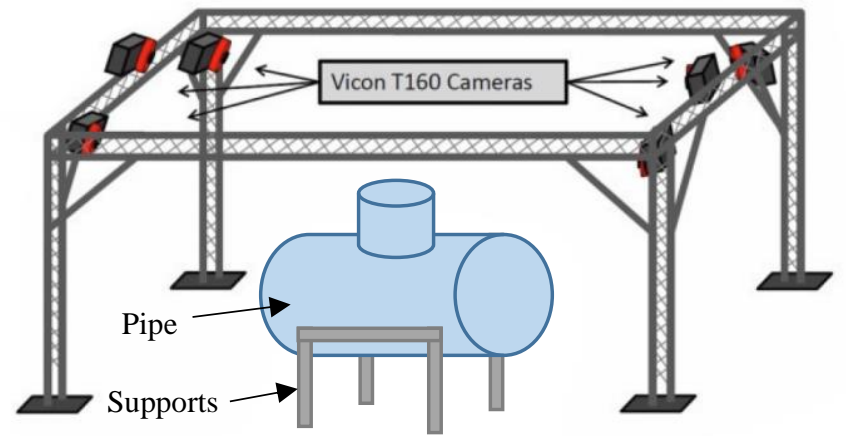

Fig. 14. Schematic of the experimental setup showing the Vicon frame and measurement volume

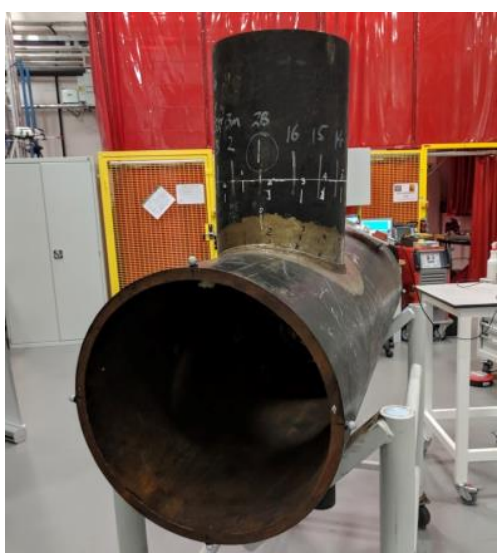

Fig. 15. Pipe with saddle weld extrusion used for the experiment and placed in the Vicon measurement volume 


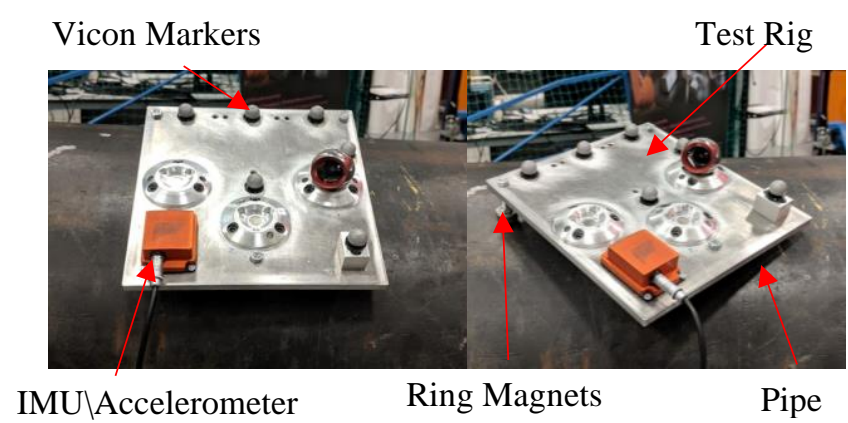

Fig. 16. Test rig setup showing the 3 points of contact with the Vicon marks and IMU/Accelerometer attached

\section{EXPERIMENTAL RESULTS}

The experimental results are shown in TABLE II and Fig. 17. These results show that the optimisation method significantly improves on the analytical approximation algorithm.

Fig. 17 and Fig. 18 show examples of the discrepancy between the algorithm and optimisation position and orientation angles calculated using the accelerometer data with the angles recorded using the Vicon system.
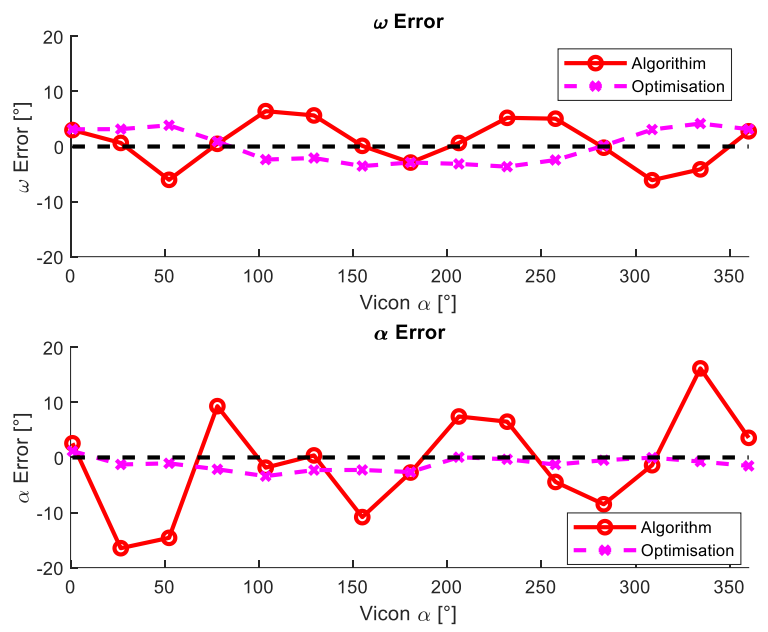

Fig. 17. Comparison between the error between the angles calculated from the accelerometer and the measured Vicon angles for $\mathrm{b}=180 \mathrm{~mm} \mathrm{l}=180 \mathrm{~mm} \mathrm{R}=$ $253.5 \mathrm{~mm}$
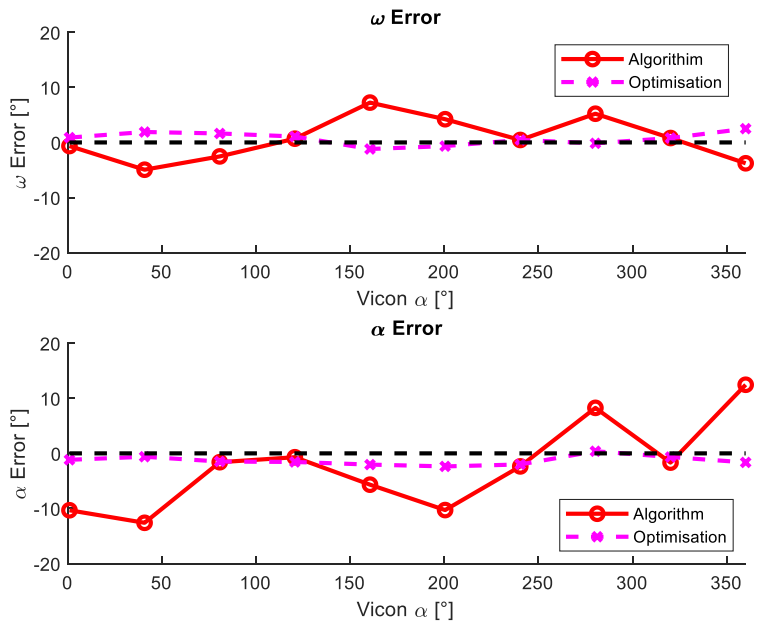

Fig. 18. Comparison between the error between the angles calculated from the accelerometer and the measured Vicon angles for $\mathrm{b}=70 \mathrm{~mm} \mathrm{l}=180 \mathrm{~mm} \mathrm{R}=$ 253.5mmTABLE II.
Max and min errors in the calculated angles compared to the Vicon data for $b$ $=180 \mathrm{~mm}$ and $\mathrm{l}=180 \mathrm{~mm}$

\begin{tabular}{ccccccc}
\hline \hline \multicolumn{7}{c}{ Error } \\
& Max & Min & Max & Min & Max & Min \\
& Inv & Inv & Para & Para & Opt & Opt \\
Alpha & $16.47^{\circ}$ & $0.37^{\circ}$ & $16.47^{\circ}$ & $0.37^{\circ}$ & $3.40^{\circ}$ & $0.04^{\circ}$ \\
Omega & $6.42^{\circ}$ & $0.12^{\circ}$ & $5.28^{\circ}$ & $0.98^{\circ}$ & $4.17^{\circ}$ & $0.14^{\circ}$ \\
\hline \hline
\end{tabular}

NB: Inv - Inverse, Para - Parametric, Opt - Optimisation

TABLE III.

Max and min errors in the calculated angles compared to the Vicon data for $\mathrm{b}$ $=70 \mathrm{~mm}$ and $\mathrm{l}=180 \mathrm{~mm}$

\section{Error}

\begin{tabular}{ccccccc}
\hline & $\begin{array}{c}\text { Max } \\
\text { Inv }\end{array}$ & $\begin{array}{c}\text { Min } \\
\text { Inv }\end{array}$ & $\begin{array}{c}\text { Max } \\
\text { Para }\end{array}$ & $\begin{array}{c}\text { Min } \\
\text { Para }\end{array}$ & $\begin{array}{c}\text { Max } \\
\text { Opt }\end{array}$ & $\begin{array}{c}\text { Min } \\
\text { Opt }\end{array}$ \\
Alpha & $12.60^{\circ}$ & $0.70^{\circ}$ & $12.60^{\circ}$ & $0.70^{\circ}$ & $2.37^{\circ}$ & $0.39^{\circ}$ \\
Omega & $7.23^{\circ}$ & $0.45^{\circ}$ & $3.56^{\circ}$ & $1.05^{\circ}$ & $2.50^{\circ}$ & $0.16^{\circ}$ \\
\hline \hline
\end{tabular}

NB: Inv - Inverse, Para - Parametric, Opt - Optimisation

\section{A. Possible Sources of Error}

There are several factors which could have affected the readings which results in the discrepancy between the measured Vicon angles and the clockface and orientation angles calculated using the accelerometer readings. These include:

- Pipe not level: The pipe may not have been perfectly flat. The test specimen used has a secondary pipe extrusion at its centre. This may cause the pipe to bend due to the weight of the added material. The pipe level varied by $3 \mathrm{~mm}$ over the total length $(1.53 \mathrm{~m})$ of the pipe.

- Floor not level: The floor which the pipe was on may not have been flat. The floor was measured using a spirit level and found to vary by $<1 \mathrm{~mm}$ over a $1 \mathrm{~m}$ distance.

- Test rig not flat: The flatness of the test rig was tested using a circular spirit level. Human error is present and therefore this may cause an error in the calculated angles. The flatness was tested with the spirit level and manually adjusted.

- Local bumps or grooves on pipe: The test piece used was an industrial pipe which had local pits and grooves. If the rig was placed on one of these the calculated angles would be affected. Ovality of the test pipe was measured using the Leica laser system to determine the cylindricality of the test pipe which showed a maximum variation in the pipe outer radius of $1.8 \mathrm{~mm}$ which represents a $0.7 \%$ ovality.

- Error in Vicon measurements: The Vicon was taken to be ground truth, however [29] showed that the error associated between the measured position and the real position was an average of $1.48 \mathrm{~mm}$ using the active calibration method employed in this work. This is in 
the same region of values of the $1.8 \mathrm{~mm}$ circumferential error recorded.

All these sources of error will accumulate, resulting in the disagreement between the calculated angles and the measured angles.

One reason the algorithm error is large compared to the Vicon measurements is because the algorithm does not consider change in pitch of the robot as it rotates. This explains why the algorithm error shows a peak error in the $\omega$ angle at approx. $45^{\circ}$ $90^{\circ} 270^{\circ}$ and $315^{\circ}$. The results show that the optimisation step corrects these inaccuracies.

\section{ERROR ANALYSIS}

There are two main sources of error which have been investigated in this work. These being the assumption that the pipe is perfectly horizontal and level with the flat ground, error in the accelerometer readings, and ovality of the pipe.

Fig: 19 shows the error associated with the calculated angles as the pipe angle is increased from $0^{\circ}$ to $5^{\circ}$ with the robot rotating $360^{\circ}$ in $\alpha$, while assuming the pipe is horizontal. The error between the simulated and optimised $\alpha$ and $\omega$ angles is then calculated. This shows that the elevation angle of the pipe is an important factor to consider, as a small pipe angle of $5^{\circ}$ can have as much as a $10^{\circ}$ error in the calculated orientation of the robot. Pipe angle appears to have a less significant effect on the $\omega$ angle as there is a maximum discrepancy of $2^{\circ}$ for a $5^{\circ}$ error in pipe angle. The alpha error appears to correspond to the pipe angle error.
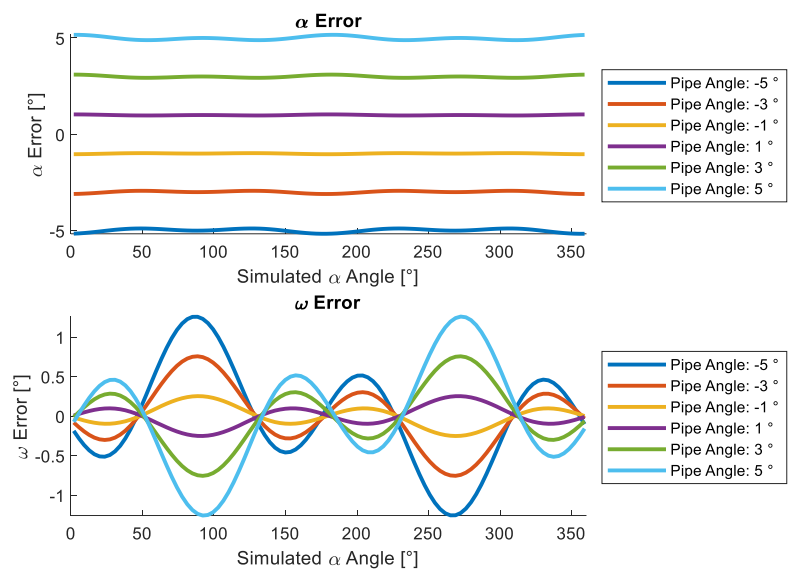

Fig: 19. Error in calculated angles with changing pipe angle

Simulations were conducted to investigate the effect of incorrect accelerometer readings in the $\mathrm{X}, \mathrm{Y}$ and $\mathrm{Z}$ directions. This error can be present in real environments in the form of random from sources such as outside vibrations from the pipe, or systematic errors in the calibration of the accelerometer. Error in terms of a percentage error in gravity was added to each of the $\mathrm{X}, \mathrm{Y}, \mathrm{Z}$ accelerometer readings. These are shown in figure Fig: 20 and shows that as error increases, the error increases. Error in the $\mathrm{X}_{\mathrm{acc}}$ and $\mathrm{Y}_{\mathrm{acc}}$ affect the $\alpha$ and $\omega$ similarly, while error in the $Z_{\text {acc }}$ mainly affects the $\omega$ angle, with minimal effect on the calculated $\alpha$ angle.,
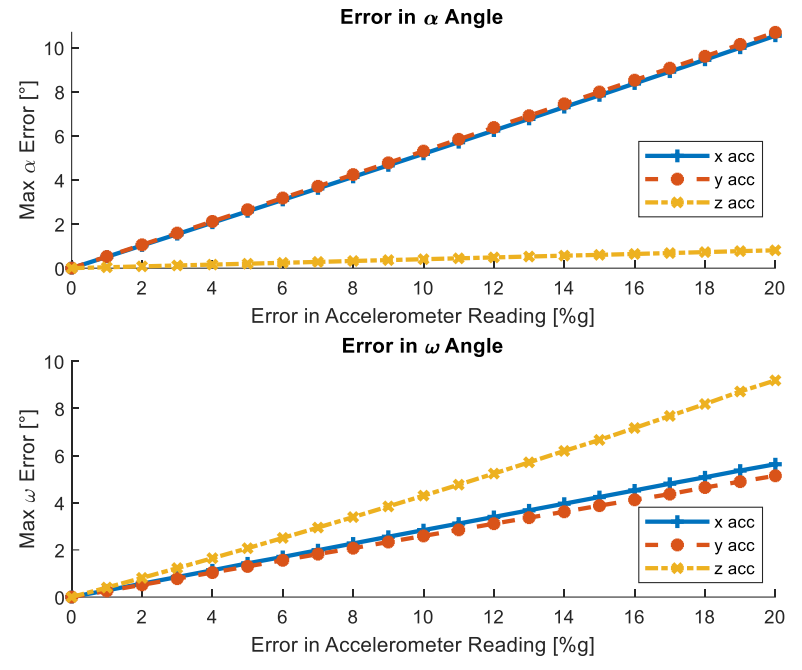

Fig: 20. Error in calculated angles as error is added to the $X, Y, Z$ acceleration readings

Another error which may be present when working with nonideal pipes is that the pipe may not be perfectly cylindrical in shape. The error associated with increasing ovality was solved numerically and is shown in Fig: 21. ASME guidelines state a tolerance of $<8 \%$ for ovality and many pipe manufactures consistently claim an ovality tolerance of $2 \%$ or less. These represent a $2^{\circ}$ and $0.5^{\circ}$ error in alpha respectively. A $0.7 \%$ ovality corresponds to a $0.2^{\circ}$ error in $\omega$.

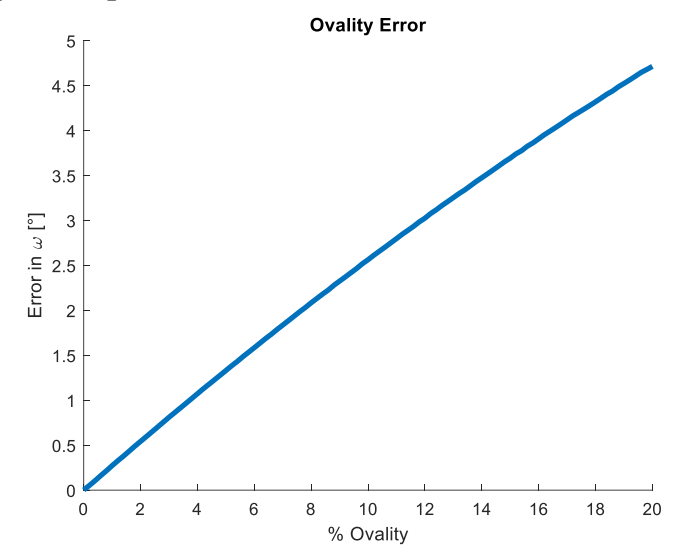

Fig: 21. Error in $\omega$ angle calculation with respect to increasing ovality of the pipe

\section{Discussion AND CONCLUSION}

Considering the sources of error, this work shows a novel method of calculating the circumferential and orientation angles of a three-wheeled robot from accelerometer sensor readings.

The algorithm plots in Fig. 17 A) and Fig. 17 B) appear to follow the patterns shown in Fig. 9 and Fig. 11 respectively. There was a maximum error in the optimisation solution observed of $\Delta \alpha=3.40^{\circ}$ and $\Delta \omega=4.17^{\circ}$. These values are within a $5^{\circ}$ error which is deemed acceptable. However, as mentioned previously, there are possible sources of error which will also be present in a real-world industrial environment which may have accumulated to realise the error shown. TABLE II and TABLE III compare the Inverse model, parametric method and optimisation solutions. The results show that there is an increase in accuracy in the $\omega$ angle when comparing the 
parametric and optimisation methods. It should be noted that there the $\alpha$ angle was not corrected in the parametric method for the reasons outlined in Section V.A.

This work presents a novel method of using accelerometer measurements to obtain absolute position and orientation measurements from an internal sensor for positioning on a pipe surface. The calculated angles rely on gravity as a constant reference, therefore the output of this will not have integral error which accumulates with time. This absolute measurement type is vital for robotic applications which need to be tracked over a long period of time and will be a necessary requirement for fully autonomous pipe inspection.

This positioning method could be improved by fusing other sensor information, such as an encoder, gyroscope or magnetometer readings for real time positioning considering historical positioning data. Kalman filters are a form of Bayesian filtering that is commonly used for random noise reduction and sensor fusion for robot positioning [9]. A Kalman filter can be used to correct encoder position measurements and prevent them from drifting over time, providing that there is an absolute measurement used for the correction step.

The experiments show that the algorithm developed calculates the positional angle $(\omega)$ and the orientation $(\alpha)$ on a horizontal pipe using only the knowledge of the robot and pipe geometry, and the output of a 3-axis accelerometer.

\section{REFERENCES}

[1] R. Singh, 'Three Decades of NDI Reliability Assessment', Karta Technologies Inc., Contract Karta3510-99-01, May 2000.

[2] P. A. Hancock and M. H. Chignell, 'Mental workload dynamics in adaptive interface design', IEEE Transactions on Systems, Man, and Cybernetics, vol. 18, no. 4, pp. 647-658, Jul. 1988.

[3] VICON, 'Motion Capture Systems', VICON. [Online]. Available: http://www.vicon.com. [Accessed: 31-May2018].

[4] 'Construction Total Stations \& GNSS'. [Online]. Available: https://leica-geosystems.com/engb/products/construction-tps-and-gnss. [Accessed: 09Aug-2018].

[5] S. F. Burch, N. J. Collett, S. Terpstra, and M. V. Hoekstra, 'M-skip: a quantitative technique for the measurement of wall loss in inaccessible components', Insight-Non-Destructive Testing and Condition Monitoring, vol. 49, no. 4, pp. 190-194, 2007.

[6] G. Dobie, S. Gareth Pierce, and G. Hayward, 'The feasibility of synthetic aperture guided wave imaging to a mobile sensor platform', NDT \& E International, vol. 58, pp. 10-17, Sep. 2013.

[7] C. N. Macleod, G. Dobie, S. G. Pierce, R. Summan, and M. Morozov, 'Machining-Based Coverage Path Planning for Automated Structural Inspection', IEEE Transactions on Automation Science and Engineering, vol. 15, no. 1, pp. 202-213, Jan. 2018.

[8] R. Siegwart, I. R. Nourbakhsh, and D. Scaramuzza, Introduction to autonomous mobile robots, 2 . ed. Cambridge, Mass.: MIT Press, 2011.
[9] H. H. S. Liu and G. K. H. Pang, 'Accelerometer for Mobile Robot Positioning', IEEE TRANSACTIONS ON INDUSTRY APPLICATIONS, vol. 37, no. 3, p. 8, 2001.

[10] P. Goel, S. I. Roumeliotis, and G. S. Sukhatme, 'Robot Localization Using Relative and Absolute Position Estimates', p. 7.

[11] L. Lou and X. Xu, 'An Approach to Improving Attitude Estimation Using Sensor Fusion for Robot Navigation', Procedia Engineering, vol. 15, pp. 5601-5605, 2011.

[12] Y. Liu, N. Noguchi, and K. Ishii, 'Attitude Angle Estimation for Agricultural Robot Navigation Based on Sensor Fusion with a low-cost IMU', IFAC Proceedings Volumes, vol. 46, no. 4, pp. 130-134, 2013.

[13] M. Kok, J. D. Hol, and T. B. Schön, 'Using Inertial Sensors for Position and Orientation Estimation', Foundations and Trends in Signal Processing, vol. 11, no. 1-2, pp. 1-153, Apr. 2017.

[14] Yun Xiaoping, E. R. Bachmann, and R. B. McGhee, 'A Simplified Quaternion-Based Algorithm for Orientation Estimation From Earth Gravity and Magnetic Field Measurements', IEEE Transactions on Instrumentation and Measurement, vol. 57, no. 3, pp. 638-650, Mar. 2008.

[15] Seong-hoon Won, N. Parnian, F. Golnaraghi, and W. Melek, 'A quaternion-based tilt angle correction method for a hand-held device using an inertial measurement unit', 2008, pp. 2971-2975.

[16] S. Cho, J. Park, and J. Lee, 'A dynamic localization algorithm for a high-speed mobile robot using indoor GPS', Robotica, vol. 30, no. 04, pp. 681-690, Jul. 2012.

[17] T. Lee, J. Shin, and D. Cho, 'Position estimation for mobile robot using in-plane 3 -axis IMU and active beacon', in 2009 IEEE International Symposium on Industrial Electronics, 2009, pp. 1956-1961.

[18] L. Lou, X. Xu, J. Cao, Z. Chen, and Y. Xu, 'Sensor fusion-based attitude estimation using low-cost MEMSIMU for mobile robot navigation', 2011, pp. 465-468.

[19] G. Welch and G. Bishop., 'An Introduction to the Kalman Filter', University of North Carolina at Chapel Hill, 1995.

[20] B. Liu, S. Cheng, and Y. Shi, 'Particle Filter Optimization: A Brief Introduction', in Advances in Swarm Intelligence, 2016, pp. 95-104.

[21] 'RMS2 | Automated Ultrasonic Corrosion Mapping Scanner | Silverwing'. [Online]. Available: https://www.silverwingndt.com/ultrasonic-testing/rms2aut-corrosion-mapping. [Accessed: 21-Mar-2017].

[22] 'Pulsed Eddy Current (PEC) Probes and Accessories', Eddyfi. [Online]. Available: http://www.eddyfi.com/pulsed-eddy-current-pecprobes/. [Accessed: 28-Mar-2019].

[23] M. Lorenz and S. Lewandowski, 'Ultrasonic multi-skip inspection at clamped saddle supports', in 18th World Conference on non-destructive Testing, 2012, pp. 1620.

[24] H. Li, 'CHAPTER III: Gröbner Bases and Basic Algebraic-Algorithmic Structures', in Noncommutative Gröbner Bases and Filtered-Graded Transfer, H. Li, Ed. Berlin, Heidelberg: Springer Berlin Heidelberg, 2002, pp. 67-90. 
[25] P. Schatte, 'Computing the Angle between Vectors', Computing, vol. 63, no. 1, pp. 93-96, Jul. 1999.

[26] V. A. Ajay, A. P. Suherlan, G. S. Soh, S. Foong, K. Wood, and K. Otto, 'Localization and Trajectory Tracking of an Autonomous Spherical Rolling Robot Using IMU and Odometry', 2015, p. V05AT08A058.

[27] 'When it has to be right | Leica Geosystems'. [Online]. Available: https://leica-geosystems.com/en-gb. [Accessed: 31-May-2018].

[28] C. N. MacLeod, R. Summan, G. Dobie, and S. G. Pierce, 'Quantifying and Improving Laser Range Data When Scanning Industrial Materials', IEEE Sensors Journal, vol. 16, no. 22, pp. 7999-8009, Nov. 2016.

[29] R. Summan et al., 'Spatial calibration of large volume photogrammetry based metrology systems', Measurement, vol. 68, pp. 189-200, May 2015.

[30] ASME-B31.1, 'ASME Code for Pressure Piping, B31 ASME B31.1-2001'. American Society of Mechancial Engineers, 2001.

[31] Železiarne Podbrezová, 'Steel Tube and Pipe Handbook’. Železiarne Podbrezová, 2009.

Alexander McGregor is currently pursuing an Engineering Doctorate with a focus on robotics for non-destructive evaluation with the Research Centre for Non-Destructive Evaluation (RCNDE)

$\mathrm{He}$ received the M.Eng in mechanical engineering with financial management from the University of Strathclyde in 2016

Gordon Dobie received the M.Eng degree in electrical and mechanical engineering from the University of Strathclyde and the $\mathrm{Ph} . \mathrm{D}$ degree from the Centre of Ultrasonic Engineering with a focus on development of reconfigurable non-contact inspection system. He is currently a Lecturer with the department of Electronic and Electrical Engineering, University of Strathclyde. He is currently with CUE, where he is involved in automated ultrasonic inspection of complex geometries.

His primary research interests include ultrasonics, NDE, automation, robotics, signal processing, computer vision and embedded systems.

Neil R. Pearson received the Ph.D. degree in MFL technology from the College of Engineering, Swansea University, Swansea, U.K., in 2015. He is the Principal Scientist with Eddyfi (U.K.) Ltd., Swansea. Since 2003, he has been involved in researching and developing magnetic flux leakage technology and automated ultrasonic testing products

Charles N. MacLeod received the M.Eng degree in electrical and mechanical engineering from the University of Strathclyde, and Ph.D. degree in automated non-destructive evaluation. He is currently a Research Fellow with the Centre for Ultrasonic Engineering.

He received the University of Strathclyde EPSRC Doctoral Prize in 2014, for investigating automated non-contact ultrasound. His research interests include robotics, sensors, electronics, mechanical fixturing and software.
Anthony Gachagan is the Director of the Centre for Ultrasonic Engineering (CUE), based in the Department of Electrical and Electronic Engineering (EEE) at Strathclyde University. He received his Ph.D. degree from the University of Strathclyde in 1996 for the development of aircoupled piezoelectric transducer technology. He has worked in the field of ultrasound for over 20 years and is the author of over 100 research publications across a broad application range including non-destructive evaluation (NDE), sonar, bioacoustics, and industrial process control. His specific research interests encompass ultrasonic transducers and arrays, array imaging processing, power ultrasound, industrial process control instrumentation, and the application of coded excitation techniques. 